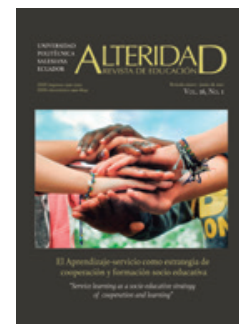

\title{
La improcedencia de estandarizar el trabajo docente: Un análisis desde Chile
}

\author{
The inadmissibility of standardizing the teaching work: An \\ analysis from Chile
}

Cristian Oyarzún-Maldonado es doctorando e investigador de la Universidad de Chile (Chile) (cristian.oyarzunm@usach.cl)

(https://orcid.org/0000-0003-481 1-8633)

(D) Rodolfo Soto-González es doctorando e investigador de la Universidad de Barcelona (España) (rodolfo.soto@usach.cl) (https://orcid.org/0000-0003-3061-6036)

Recibido: 2019-03-15 / Revisado: 2020-10-17 / Aceptado: 2020-12-02 / Publicado: 2021-01-01

\section{Resumen}

Cada vez, más países implementan políticas de rendición de cuentas y estandarización como solución a los problemas de equidad y calidad educativa. Este artículo examina la pertinencia de diseñar el trabajo docente con base en diferentes mecanismos de estandarización. Para ello, se utiliza la propuesta de Henry Mintzberg, una teoría de comportamiento organizacional que plantea mecanismos de coordinación referidos a distintas formas de organizar el trabajo según sus características y objetivos. Con este marco teórico se analiza el modelo escolar chileno, que sobresale por el uso de la estandarización para regular procesos y resultados. El análisis confirma la hegemonía de estos mecanismos de estandarización mediante distintos dispositivos evaluativos y prescriptivos de la política escolar, así como también permite afirmar su improcedencia por efecto de diversas externalidades negativas empíricamente sustentadas. A partir de este examen se discute la pertinencia de otros mecanismos de coordinación para el diseño de la política escolar, proponiéndose un modelo que prevalezca en formas regulatorias ligadas con el desarrollo de habilidades situadas, el trabajo colaborativo y la formación en valores socioeducativos.

Descriptores: Modelo escolar chileno, políticas escolares, trabajo docente, estandarización de procesos, estandarización de resultados, comportamiento organizacional.

\section{Abstract}

Every day, more countries implement reimbursement and standardization policies as a way to solve problems related to equity and quality education. This article examines the design appropriateness of teaching work, based on different standardization mechanisms. Thus, the Henry Mintzberg proposal is used, an organizational theory behavior that proposes coordination mechanisms regarding different ways of organizing work according to its characteristics and objectives. Using this theoretical framework, the Chilean school model is analyzed, which stands out using standardization to regulate processes and results. The analysis confirms the hegemony of these standardization mechanisms using different evaluative and prescriptive devices of the school policy, as well as allows to affirm their inadmissibility because of diverse empirically supported negative externalities. Emanating of mentioned above, the appropriateness of other coordination mechanisms for the design of school policy is discussed, proposing a model that prevails in regulatory forms linked to the development of situated skills, collaborative work and training in socioeducational values.

Keywords: Chilean school model, school policies, teaching work, standardization of processes, standardization of results, organizational behavior.

Forma sugerida de citar: Oyarzún-Maldonado, C., \& Soto-González, R. (2021). La improcedencia de estandarizar el trabajo docente: Un análisis desde Chile. Alteridad, 16(1), 105-116. https://doi.org/10.17163/alt.v16n1.2021.08 


\section{Introducción}

Ante el desafío de mejorar la calidad y equidad de los sistemas escolares contemporáneos, uno de los modelos de gobernanza de mayor auge en la actual agenda educativa global es el New Public Management (NPM) que, con distintas adaptaciones locales, ha sido implementado en diversos países de Asia, Europa, Latinoamérica y Norteamérica (Bezes et al., 2012; Hall et al., 2015; Holloway et al., 2017; Maroy \& Pons, 2019; Maroy et al., 2016; Normand et al., 2018). Dicho modelo se basa en la transferencia de teorías y técnicas de gestión desde la empresa privada a organizaciones tradicionalmente públicas (Ball \& Youdell, 2008) como, por ejemplo, las escuelas. Este marco se concretiza en un conjunto de medidas que pueden incluir provisión mediante cuasi-mercados, descentralización administrativa, convenios de desempeño, estandarización de prácticas y resultados, accountability con consecuencias de alto riesgo, pago por el logro de metas, entre otras (Anderson \& Cohen, 2015; Carvalho \& Normand, 2018; Gleeson \& Knights, 2015; Verger \& Normand, 2015).

Lejos de generar consensos y adhesión irrestricta, la aplicación del NPM en el campo educativo provoca intensos debates y cuestionamientos acerca de su capacidad para propender hacia el mejoramiento escolar (Maroy \& Pons, 2019; Normand et al., 2018). Así, la literatura internacional ha concentrado particular interés en aquellos dispositivos que estandarizan resultados y prácticas pedagógicas, así como en las externalidades negativas que conllevan para el trabajo docente. $\mathrm{Al}$ respecto, se ha sostenido que la utilización de pruebas estandarizadas de medición de aprendizajes a gran escala vinculadas a consecuencias provoca un reduccionismo curricular y una sobrevaloración de los resultados, convirtiéndose estos en el propósito unívoco de la enseñanza (Chan, 2010; Darling-Hammond, 2014; Luengo-Navas, \& Saura-Casanova, 2013). A su vez, se han reportado hallazgos acerca de la estandarización de las labores del profesorado, los cuales advierten de profundos cambios en la labor cotidiana que se traducen en fenómenos de performatividad (Ball et al., 2013), emasculación de saberes locales frente a las prescripciones de la política (Herr, 2015) y malestar psicológico por efecto de una intensificación del trabajo para lograr su adecuación a dichas prescripciones (Tsang \& Kwong, 2016; Viera \& Oliveira, 2013).

En este contexto general, el presente artículo expone un análisis teórico respecto de las dificultades que conlleva aplicar mecanismos de estandarización propios del NPM en el trabajo docente y su inadecuación metodológica para regular las prácticas pedagógicas. Con este propósito, se utiliza como marco analítico la teoría de Henry Mintzberg, una teoría de comportamiento organizacional que plantea mecanismos de coordinación referidos a seis formas de operacionalizar, verificar y articular las actividades de una organización, los cuales varían según la naturaleza y objetivos del trabajo (Mintzberg, 1980; 1984). A través de los mecanismos de coordinación, esta teoría aborda profusamente la estandarización por resultados y procesos, proporcionando argumentos respecto de su conexión a actividades laborales específicas. Por ende, estos planteamientos conceptuales permiten analizar la pertinencia o improcedencia de estas formas de coordinación aplicadas al trabajo docente.

Por su parte, el objeto de este análisis se concentrará en la política escolar de Chile, caso nacional calificado como extremo en cuanto a la adopción de políticas promercado y dispositivos basados en el NPM, y que sobresale por el uso de mecanismos de estandarización tanto a nivel de la gestión escolar como del trabajo docente (Assaél \& Cornejo, 2018; Falabella, 2015; Verger et al., 2016). Puntualmente, se releva la estandarización de resultados ejercida por medio del SIMCE, ${ }^{1}$ que no solo evalúa los niveles de aprendizaje alcanzados mediante pruebas estandarizadas de alcance nacional, sino que además constituye la principal fuente de datos utilizada para la elaboración de un ranking de desempeño ${ }^{2}$ asociado a castigos que se aplican sobre las escue- 
las (Assaél et al., 2018; Carrasco, 2013; Pino et al., 2016). Mientras que se identifica una intensa estandarización de las prácticas pedagógicas, observable en instrumentos oficiales que sistematizan "mejores prácticas", entre los que puede nombrarse el Manual de la Buena Enseñanza (MBE) que opera de modo prescriptivo al sustentar un Sistema Nacional de Evaluación Docente con consecuencias de alto riesgo (Ávalos, 2017; Cavieres \& Apple, 2016; Cornejo et al., 2015; Fardella, 2013; Sisto, 2012). La temática adquiere una relevancia adicional para el contexto latinoamericano debido a la creciente difusión de modelos de privatización educativa en la región (Verger et al., 2017) y, en particular, de modelos basados en la estandarización (Internacional de la Educación para América Latina, 2015).

Considerando todo lo anteriormente expuesto, este artículo se estructura en tres apartados principales. En el primer apartado se ofrece una descripción conceptual de los mecanismos de coordinación postulados en la teoría de Mintzberg. En segundo lugar, se analiza la correspondencia de la estandarización de procesos y de resultados con las formas de estandarización aplicadas en el contexto de la política escolar chilena, así como también se plantean cuestionamientos sobre la aplicación de este tipo de estandarizaciones en el trabajo docente. En el tercer apartado se proponen otros mecanismos de coordinación que, a partir del análisis, resultan más concernientes a la actividad pedagógica. Finalmente, en las conclusiones se expone una síntesis del análisis efectuado y sus implicancias para el diseño de la política.

\section{Los mecanismos de coordina- ción de Henry Mintzberg}

Todas las organizaciones requieren diseñar y realizar un cierto número de tareas específicas para alcanzar sus objetivos. Por ello, toda actividad productiva requiere de un proceso o una secuencia de acciones interdependientes que poseen un comienzo y un final, las cuales pueden ser regu- ladas mediante mecanismos de coordinación (Pucheu, 2013; Schmidt, 2006). Como se anticipó, los mecanismos de coordinación son diversas formas de sistematizar, verificar y articular el trabajo, que se utilizan para diseñar y garantizar el éxito de un proceso productivo. Ergo, se les considera elementos fundamentales en la estructura de una organización, en tanto operan como el aglutinante que la mantiene unida y comunicada. Siguiendo los postulados de la cibernética, también pueden ser entendidos en términos de formas de control, en la medida que - coordinación y control- se orientan a comprobar la calidad de las acciones ejecutadas (Mintzberg, 1992; 1993).

Henry Mintzberg (1980) propone seis mecanismos de coordinación:

El primero se denomina ajuste mutuo y consiste en obtener la coordinación del trabajo mediante la simple comunicación informal en interacciones horizontales. Este medio de coordinación es característico en organizaciones poco estructuradas, donde la gente trabaja en estrecha colaboración y con base en relaciones informales. Asimismo, suele emplearse en organizaciones de alta especialización, pues funciona ante circunstancias de extrema complejidad e incertidumbre que requieren elaborar respuestas innovadoras (Mintzberg, 1984).

El segundo es la supervisión directa, que consigue la coordinación endosando la responsabilidad a una persona sobre el trabajo de los demás, verticalizándose las relaciones laborales mediante instrucciones y observancia directa (Mintzberg, 1984).

El tercer mecanismo es la estandarización de procesos, referida a una forma de coordinación relacionada con un programa de trabajo que prescribe secuencias de procesos o prácticas, teniendo por consecuencia la reducción de la necesidad de una comunicación continuada y presencial. Esta coordinación es determinada $a$ priori, es decir, antes que se realicen las tareas laborales, pues los procesos de trabajo, tanto los inputs - habilidades y conocimientos- como 
los outputs - acciones-pueden confeccionarse en una normativa predeterminada o manual de procedimientos (Mintzberg, 1984).

El cuarto término es la estandarización de resultados, que tiene por finalidad normalizar los resultados mediante su prescripción, focalizándose únicamente en el rendimiento esperado (Mintzberg, 1984).

Como quinto mecanismo, se tiene la estandarización de habilidades, que constituye la forma de coordinación más apropiada en tareas complejas, las cuales no pueden emplear las dos formas anteriores de estandarización (Pucheu, 2013). Con más detalle, esta modalidad de estandarización trata sobre la especificación de las habilidades y conocimientos requeridos para la realización de un trabajo. En consecuencia, la estandarización de habilidades logra por vía indirecta y orgánica lo que las otras normalizaciones — procesos y resultados- intentan conseguir de forma exógena y prescriptiva (Mintzberg, 1984).

Finalmente, se encuentra la estandarización de criterios, que concibe la coordinación a partir de la transmisión de un sistema de creencias y significados compartido entre los integrantes de la organización, es decir, la construcción de una cultura organizacional (Mintzberg, 1984).

Si bien ninguna organización basa su funcionamiento en un único mecanismo de coordinación, sino que estos tienen ocurrencia simultánea, es habitual que los objetivos organizacionales y los parámetros de diseño del trabajo ejerzan un predominio a favor de unos mecanismos por sobre los demás. En este sentido, siguiendo la noción de predominancia, Mintzberg (1984) identifica una tipología de organización cuyo funcionamiento se basa preminentemente en la estandarización de procesos y de resultados, centrales en el contexto del presente análisis.

Efectivamente, existen organizaciones que se desenvuelven en diversos sectores productivos y de servicios, empero se caracterizan por realizar una labor operativa que es rutinaria y con procesos altamente estandarizados, esta es la denominada burocracia mecánica. En esta confi- guración, la tecnoestructura ${ }^{3}$ ejerce su influencia prescribiendo los procesos de trabajo mediante una descentralización limitada de carácter horizontal y generando una estructura orientada al orden y la repetición de procesos para alcanzar resultados también prescritos (Mintzberg, 1980). La burocracia mecánica es efectiva en condiciones donde la tarea y el entorno suelen ser estables y simples. Por el contrario, estos sistemas de control centralizados generan organizaciones que, al tratar con circunstancias variables, se vuelven lentas e ineficaces. Esto se hace patente en actividades donde se buscar proveer servicios personales o de transferencia de capacidades, pues estas actividades requieren respuestas particulares a las necesidades de cada usuario (Pucheu, 2013).

\section{La estandarización de proce- sos y resultados en el trabajo docente: El caso de Chile y sus cuestionamientos}

En un sistema escolar de amplitud nacional es posible que tengan cabida todos los mecanismos de coordinación postulados por Mintzberg. Sin embargo, en el caso chileno la adopción de dispositivos del NPM ha fortalecido la aplicación de consecuencias en forma de incentivos y amenazas de despido o cierre ante el no cumplimiento de las prácticas y los resultados estandarizados (Oyarzún et al., 2019). Por lo anterior, incluso si se presenta coexistencia de múltiples formas de coordinación, son los mecanismos de estandarización de procesos y resultados aquellos que consiguen mayor centralidad en el sistema escolar chileno. En este apartado se analizarán sintéticamente cómo estos dos mecanismos condicionan la labor docente, junto con los cuestionamientos que se desprenden a partir de tal examen.

Para comprender la manera en que la estandarización de procesos se presenta normativamente en el quehacer de los profesores desde la óptica de Mintzberg (1984), es necesario tomar nota que este autor sugiere para la organización 
de las escuelas la pertinencia de la estandarización de habilidades. Las configuraciones estructurales que resultan de la primacía de alguno de estos mecanismos de estandarización, ya sea de procesos, resultados o habilidades, difieren fundamentalmente en cuanto a la autonomía conferida al personal del núcleo de operaciones, es decir, los profesores que trabajan en las aulas. En términos simples, ante la estandarización de habilidades la autonomía de los docentes se alza, mientras que frente a la estandarización de procesos o de resultados decrece, manteniéndose el poder en las prescripciones externas de los instrumentos de la política.

En Chile, las disposiciones legales han asegurado que los docentes sean posicionados como ejecutores de planes, bases curriculares y marcos normativos ajenos a la escuela, elaborados sin su participación y, por tanto, reduciendo su autonomía (Cornejo et al., 2015; Fernández et al., 2016; Sisto, 2012). Los artefactos y dispositivos que formalizan el contenido de la práctica pedagógica de manera más directa comprenden las Bases Curriculares obligatorias, los textos escolares de distribución estatal, el MBE y diversos mecanismos de accountability con consecuencias de alto riesgo, aplicados tanto a nivel de los docentes como de la escuela (Assaél et al., 2018).

Como ejemplo de lo anterior, se tiene que a nivel individual el desempeño de los docentes está vinculado a un Sistema Nacional de Evaluación que deriva en consecuencias referidas a incentivos - bonificaciones individuales $\mathrm{y}$ acceso a instancias de perfeccionamiento- $y$ sanciones - posibilidades de despido luego de dos evaluaciones continuadas en nivel insuficiente, categoría de más bajo desempeño-(Bonifaz, 2011). Esta evaluación se basa en cuatro instrumentos que miden el ajuste a estándares o indicadores de desempeño contenidos en el MBE, que incluyen la irrestricta aplicación de las bases curriculares y de las prácticas pedagógicas preestablecidas (Taut \& Sun, 2014). A pesar de las modificaciones realizadas mediante la reciente Ley de Desarrollo Profesional Docente del 2016, varios autores sostienen que, en este ámbito, el sistema evaluativo conserva una modalidad gerencialista y basada, predominantemente, en una accountability individual de altas consecuencias (Assaél \& Cornejo, 2018; Ávalos, 2017; Ruffinelli, 2015). Por su parte, a nivel organizacional o de la escuela, el modelo de gestión escolar - fuertemente influenciado por el NPM- establece sistemas de contractualización vertical en que las escuelas suscriben convenios para el cumplimiento de estándares de gestión y resultados cuya inobservancia implica eventuales sanciones, lógica de funcionamiento que fue instalada con la promulgación de la Ley de Subvención Escolar Preferencial (SEP) del 2008 (Oyarzún et al., 2019). A partir de este hito, las escuelas son clasificadas según su desempeño — como se anticipó, el sistema de clasificación fue perfeccionando- de manera tal que un bajo rendimiento sostenido podría implicar el cierre de la organización escolar (Parcerisa \& Falabella, 2017).

Por sí misma, la existencia de estos artefactos y dispositivos no permite suponer que sean tratados como condicionantes de la estandarización. No obstante, en el caso chileno, dichos artefactos y dispositivos son aplicados mediante un accountability de altas consecuencias tanto a nivel organizacional como individual. Esto configura un sistema fuertemente prescriptivo que instituye la estandarización como principal mecanismo de coordinación y, por ende, como objetivo central del trabajo docente. Particular trascendencia adquiere la estandarización de resultados pues, principalmente, son los resultados obtenidos por los estudiantes en el SIMCE los que determinan, en mayor medida, las categorías de clasificación. Sumado a esto, tales resultados son publicados para ser revisados por las familias, agregando presión social y robusteciendo la centralidad de este tipo de mecanismo de coordinación. Las investigaciones y experiencias desarrolladas en Chile reportan que tales disposiciones confinan el trabajo docente hacia prácticas no esperadas como la pérdida de instancias de trabajo colaborativo (Assaél et al., 2012; Assaél et al., 2014) 
y el entrenamiento para las evaluaciones estandarizadas, llevando a la desvalorización de otras áreas de conocimiento (Pino et al., 2016; Reyes \& Akkari, 2017; Weinstein et al., 2016). De este modo, la desprofesionalización estriba en que se espera el acatamiento de estos instrumentos y evaluaciones externas en condiciones donde el tiempo lectivo es insuficiente $y$, además, se exige responsabilización de un alto volumen de trabajo administrativo, destinado únicamente a la producción de evidencias para rendir cuentas (Assaél et al., 2012; Braslavsky, 1999; Fardella, 2013; Rojas \& Leyton, 2014).

Entonces, ¿cuál sería el sentido de estandarizar los procesos y resultados de un trabajo? Según Mintzberg, las actividades ocupacionales se estandarizan para reducir su variabilidad, operar en condiciones calificadas como libres de incertidumbres y, de esta manera, garantizar su predicción; así como también para responder a un deseo arbitrario de orden, ejercer control sobre el comportamiento, concentrar el poder en la tecnoestructura y/o percibir al trabajador como una pieza intercambiable. Empero, en la pedagogía la variabilidad y la diversidad son la regla $y$, por ende, estructurar la práctica pedagógica a un estudiante "promedio" e inexistente no hace más que promover la exclusión y generar un servicio educativo que aborda inoportunamente las necesidades de los educandos (Meyer et al., 2014).

Es más, el propio Mintzberg $(1980 ; 1984)$ afirma que el trabajo profesional no puede ser controlado debido a su alta complejidad y multiplicidad de resultados posibles, siendo improcedente su estandarización por procesos o resultados. Así, la estandarización de procesos y resultados aplicada al trabajo profesional de los docentes tiene varios efectos perversos: a) la obediencia a las normas por parte de los profesionales se transforma en un fin en sí mismo; b) se vuelve inefectivo dado que el operario profesional pierde control sobre el trabajo complejo; c) reduce los procesos analíticos subyacentes al trabajo profesional; d) desequilibran la relación entre profesional-cliente (aquí profesor-estu- diante) al deshacer el contacto libre y personal; e) reduce la innovación; y f) aumenta la pasividad del profesional.

Finalmente, es pertinente examinar el papel de la supervisión directa en tanto mecanismo de coordinación que, en el contexto del sistema chileno, aparece subsumido a la supremacía de la estandarización por procesos y resultados. Si bien el discurso de la política actual atribuye a los directores e integrantes del equipo directivo un rol trascedente como "líderes pedagógicos", en su labor cotidiana tienen como principal preocupación asegurar que sus escuelas avancen en las categorías de desempeño mediante mejores resultados en el SIMCE y asegurar fuentes de financiamiento suficientes en función del sistema de subvención per cápita (Montecinos et al., 2015; Weinstein et al., 2016). En consecuencia, siguiendo la evidencia empírica sobre el caso chileno, algunas de las secuelas resultantes que implican decisiones directivas son: a) la aplicación de medidas de selección para mejorar la composición socioeconómica y cognitiva del estudiantado (Carrasco et al., 2017; Weinstein et al., 2016); b) implementación de medidas de discriminación y expulsión (Carrasco \& Fromm, $2016)$; c) que las escuelas de menor nivel socioeconómico concentren a estudiantes con discapacidad intelectual (Fundación Chile, 2013; Rosas \& Santa Cruz, 2013); y d) concentrar la enseñanza en las áreas evaluadas, apartándose de una concepción integral de la enseñanza (Assaél et al., 2018; Reyes \& Akkari, 2017).

\section{Discutiendo "los otros" meca- nismos de coordinación para el caso de Chile}

Como se señaló, Mintzberg asevera que la estandarización de procesos y resultados no son mecanismos adecuados para coordinar las prácticas pedagógicas (Mintzberg, 1984; Pucheu, 2013). Concretamente, este autor asumió que la estandarización de habilidades podía considerarse como un dispositivo que se adecuaba 
de mejor manera a la complejidad inherente de la situación educativa. Organizacionalmente, el resultado de priorizar este mecanismo conlleva la emergencia de burocracias profesionales, caracterizadas por un conjunto de profesionales que disponen de autonomía, pero que no necesariamente colaboran entre sí.

Siguiendo a Mintzberg (1984), los problemas comunes que surgen en la burocracia profesional incluyen: a) dificultad en la coordinación de los profesionales; b) rencillas a raíz de intentar comprender contingencias fuera de las categorías de conocimiento que comparten; c) dificultad para abordar profesionales carentes de ética, dado que las habilidades, por normalizadas que estén, requieren un juicio considerable; d) dificultad para abordar profesionales incompetentes que no quieren actualizar sus conocimientos; e) desatención de las necesidades de la organización, pues no se perciben como parte de un equipo; f) no adecuarse a las necesidades de los usuarios para dar lugar, únicamente, a lo que saben o quieren hacer los profesionales; g) incapacidad para innovar.

En esta línea, Braslavsky (1999) cita a Mintzberg, postulando que la teoría del último permite comprender, desde una mirada histórica, el estado de profesionalización que existía antes de la actual desprofesionalización del magisterio. Esta autora propone que, más que buscar profesionalizar o volver a la situación anterior, es necesario re-profesionalizar o reinventar la profesión docente para el siglo XXI. Este proceso debería considerar, principalmente, habilidades que permitan tanto un mejor desempeño en las problemáticas y situaciones coyunturales como una mayor participación en la reinvención de la escuela y los sistemas educativos.

Siguiendo con la mismalógica, Carbonneau y Hétu (2005) refieren que cada maestro debe enfrentar situaciones inéditas y, para las cuales, deberá elaborar una respuesta singular, pues ante los problemas educativos no existen soluciones universales. Es así como se requieren competencias para analizar críticamente las prácticas, tomando en cuenta múltiples modelos de acción (Carbonneau \& Hétu, 2005), cuerpos teóricos y normas (Altet, 2005) y, por supuesto, las necesidades y particularidades de los estudiantes en su contexto (Coll, 2016). Por ello, los marcos normativos que prescriben la práctica docente de manera genérica en Chile, por ejemplo, el MBE, pueden ser útiles como uno más entre diversos modelos o enfoques pedagógicos.

Entonces, la formación profesional depende de un análisis reflexivo efectuado con pares docentes y centrado en las prácticas pedagógicas cotidianas, cuyo resultado producirá saberes con base en la acción (Altet, 2005). Por tanto, la colaboración es esencial para impulsar procesos de innovación y mejora educativa desde una reflexión compartida (Butler \& Schnellert, 2012). Desde el modelo de Mintzberg, es posible asimilar el mecanismo denominado ajuste mutuo con la dinámica del trabajo colaborativo. Efectivamente, este teórico postula que precisamente este mecanismo permite una mejor adaptación a situaciones complejas y particulares y, además, favorece la innovación.

En este escenario, las comunidades de aprendizaje resultantes del fortalecimiento del ajuste mutuo pueden favorecer la discusión de aspectos técnicos, pero también de valores y propósitos, siendo esencial una diversidad de voces (Philpott, 2018). En consecuencia, los espacios reflexivos y colaborativos pueden incluir a docentes y, además, a otros actores educativos e integrantes de la comunidad educativa, dando paso a acuerdos comunes bajo un razonamiento democrático. Es así como también resultaría necesario la estandarización de criterios, formulada por Mintzberg, que aporta al desarrollo de culturas organizacionales, pero desde una lógica inductiva, es decir, a partir del diálogo constructivo entre los distintos actores sociales que conforman una comunidad. El desarrollo conjunto de ambos mecanismos señalados — ajuste mutuo y estandarización de criterios-podría inducir a la generación de habilidades profesionales, pero ya no a partir de la lógica del estándar emana- 
do de una matriz centralizada, sino desde una perspectiva situada y focalizada que responda a la diversidad y condición siempre cambiante y contextual de la enseñanza.

Sin perjuicio de lo anterior, la aplicación y desarrollo de formas de trabajo más afines a la pedagogía también depende de condiciones estructurales. En este sentido, se identifican tres aspectos críticos para la ocurrencia de un trabajo colaborativo, innovador y basado en conocimientos situados. Como primer punto, las prácticas de gestión promovidas desde la política que pretenden instalar dinámicas colaborativas y reflexivas a nivel de la escuela son aun incipientes, ${ }^{4}$ en función de lo cual podrían carecer de una utilización genuina por parte de los actores educativos ante la presión que ejercen los dispositivos del NPM, asimismo la exigencia obligatoria vía decretos o normativas no asegura su aparición ni permanencia. Al respecto, se identifica la pertinencia de desactivar los mecanismos sancionatorios que otorgan carácter prescriptivo a muchas instancias impulsadas desde la política escolar. En segundo lugar, es necesaria la concesión de espacios temporales que favorezcan la realización de formas de trabajo colaborativo, como las comunidades de aprendizaje. Este aspecto parece aún insuficiente, pues, aun considerando los cambios introducidos con la Ley de Desarrollo Profesional Docente, el tiempo no lectivo para la preparación de clases alcanza sólo al 35\% en 2019 (OCDE, 2017). En tercer lugar, otro aspecto refiere a revertir la escasa confianza depositada en los educadores, sus capacidades y conocimientos locales (Carrasco, 2013; Sisto, 2012). En relación con esto, la evaluación individual refrendada en la Ley de Desarrollo Profesional Docente se visualiza como un dispositivo gerencialista (Ávalos, 2017; Ruffinelli, 2016) que externaliza la categorización de "buenos" y "malos" educadores mediante el juicio de evaluadores externos con base en un sistema uniforme de estándares. Esto no solo ejerce un impulso en contra del trabajo colaborativo, sino que también evidencia desconfianza en las capacidades y el juicio profesional de los maestros.

\section{Consideraciones finales}

Los planteamientos revisados previamente permiten comprender que las aportaciones teóricas de Mintzberg se distancian de la estructuración normativa del sistema escolar chileno, en particular respecto de las prácticas pedagógicas y de la gestión escolar. Con más detalle, el discurso actual con evidente acervo tecnocrático y estandarizador que demanda la aplicación de dispositivos y tecnologías del NPM, asumidas "apriorísticamente" como efectivas (Verger \& Normand, 2015), genera una serie de efectos perversos o externalidades negativas. Es así como se posibilita que la adscripción a las prescripciones se constituya en un fin en sí mismo o que los procesos analíticos subyacentes al trabajo pedagógico se reduzcan de manera drástica, entre otras. En otras palabras, los procesos de estandarización de procesos y resultados son improcedentes ante la dinámica pedagógica y, paradojalmente, tienden a burocratizar el trabajo docente en vez de hacerlo más efectivo.

En conclusión, la hegemonía de mecanismos de estandarización por procesos y resultados que requieren condiciones de regularidad y simpleza es infructuosa y perjudicial, siendo necesarias otras formas de regular este tipo de trabajo. Por consiguiente, como corolario de este análisis y pensando en el diseño del trabajo docente, se propone considerar con mayor predominancia otros mecanismos de coordinación más afines a la labor pedagógica, entre los que se identifican: a) la estandarización de habilidades: competencias críticas y reflexivas que incluyan múltiples conocimientos teóricos y prácticos; b) el ajuste mutuo: facilitación de espacios de colaboración y comunidades de aprendizaje; y c) la estandarización de criterios: acuerdos valóricos generados inductivamente desde los centros escolares.

De este modo, la clasificación de mecanismos de coordinación plateada por Mintzberg hace varias décadas en el campo organizacional constituye un marco teórico atingente para comprender las diversas unidades y dinámicas 
que estructuran y regulan un sistema escolar. Puntualmente, a través de este ya clásico lente en el ámbito corporativo, es posible identificar múltiples problemáticas relacionadas con la forma de diseñar el trabajo docente en Chile. Inclusive, a lo largo de estas páginas hemos reflexionado, desde la perspectiva elegida, respecto de posibles líneas de transformación para la optimización del sistema escolar analizado.

\section{Notas}

1. Sistema Nacional de Medición de la Calidad Educativa: evaluación estandarizada del aprendizaje en las asignaturas de Lenguaje y Comunicación; Matemática; Ciencias Naturales; Historia, Geografía y Ciencias Sociales e Inglés. Las pruebas del SIMCE se aplican censalmente en $2^{\circ}, 4^{\circ}, 6^{\circ}, 8^{\circ}$ básico, y II medio, siguiendo un Plan de Evaluaciones (Fuente: https://bit. ly/36tDuJo).

2. La Ley 20.529 que crea el Sistema de Aseguramiento de la Calidad de la Educación (2011), fija cuatro categorías de desempeño: insuficiente, medio bajo, medio y alto. Además, en su artículo 31 tipifica las condiciones que podrían implicar la pérdida del Reconocimiento oficial para una escuela que permanece, a lo menos, cuatro años en categoría de desempeño insuficiente.

3. Grupo de tecnócratas encargados del diseño del trabajo.

4. Por ejemplo, las horas para trabajo colaborativo entre profesores de aula regular y profesionales del Programa de Integración escolar para la atención de estudiantes con necesidades educativas especiales (Fuente: https://bit.ly/391xiz0).

\section{Referencias bibliográficas}

Altet, M. (2005). La competencia del maestro profesional o la importancia de analizar las prácticas. En L. Paquay, M. Altet, E. Charlier, \& P. Perrenoud (Coords.), La formación profesional del maestro. Estrategias y competencias (pp. 33-54). Fondo de Cultura Económica.

Anderson, G., \& Cohen, M.I. (2015). Redesigning the identities of teachers and leaders: A framework for studying new professionalism and educator resistance. Education Policy Analysis Archives, 23(85). http://dx.doi.org/10.14507/epaa.v23.2086

Assaél, J., \& Cornejo, R. (2018). Work regulations and teacher subjectivity in a context of standardization and Accountability Policies in Chile. In R. Normand, M. Liu, L. Carvalho, D. Andrade, \& L. Levasseur (Eds.), Education Policies and the Restructuring of the Educational Profession, Global and Comparative Perspectives (pp. 245-257). Springer.

Assaél, J., Albornoz, N., \& Caro, M. (2018). Estandarización educativa en Chile: tensiones y consecuencias para el trabajo docente. Educação Unisinos, 22(1), 83-90.

Assaél, J., Acuña, M., Contreras, P., \& Corbalán, F. (2014). Transformaciones en la cultura escolar en el marco de la implementación de políticas de accountability en Chile. Un estudio etnográfico en dos escuelas clasificadas en recuperación. Estudios Pedagógicos, 40(2), 7-26.

http://dx.doi.org/10.4067/S0718-07052014000300001

Assaél, J., Contreras, P., Corbalán, F., Palma, E., Campos, J., Sisto, V., \& Redondo, J. (2012). Ley SEP en escuelas municipales emergentes: ¿cambios en la identidad docente? Revista de Pedagogía Crítica, 11, 219-228. http://dx.doi. org/10.25074/07195532.11.453

Ávalos, B. (2017). Teacher evaluation in Chile: Highlights and complexities in 13 of experience. Teachers and Teaching, 24(3), 297-311. https://doi.org/10.1080/13540602.2017.1388228

Ball, S., \& Youdell, D. (2008). La privatización encubierta en la educación pública. Instituto de Educación, Universidad de Londres.

Ball, S., Bailey, P., Mena, P., del Monte, P., Santori, D., Tseng, C., Young, H., \& Olmedo, A. (2013). A constituição da subjetividade docente no Brasil: um contexto global. Revista Educação em Questão, 46(32), 9-36.

https://doi.org/10.21680/1981-1802.2013v46n32ID5114

Bezes, P., Demazière, D., Le Bianic, T., Paradeise, C., Normand, R., Benamouzig, D. Pierru, F., \& Evetts, J. (2012). New public management and professionals in the public sector. What new patterns beyond opposition? Sociologie du travail, 54, 1-52. http://dx.doi.org/10.1016/j.soctra.2012.07.001

Braslavsky, C. (1999). Bases, orientaciones y criterios para el diseño de programas de formación de profesores. Revista Iberoamericana de Educación, 19, 13-50. https://doi.org/10.35362/rie1901054

Bonifaz, R. (2011). Capítulo 1: Origen de la evaluación docente y su conexión con las políticas públicas en educación. En J. Manzi, J., R. 
González, \& Y. Sun (Eds.), La evaluación docente en Chile (pp. 13-32). MIDE UC.

Butler, D.L., \& Schnellert, L. (2012). Collaborative inquiry in teacher professional development. Teaching and Teacher, 28(8), 1206-1220. https://doi.org/10.1016/j.tate.2012.07.009

Carbonneau, M., \& Hétu, J. (2005). La formación práctica de los maestros y el nacimiento de una inteligencia profesional. En L. Paquay, M. Altet, E. Charlier, \& P. Perrenoud (Coords.), La formación profesional del maestro. Estrategias y competencias (107-138). Fondo de Cultura Económica.

Carrasco, A. (2013). Mecanismos performativos de la institucionalidad educativa en Chile: Pasos hacia un nuevo sujeto cultural. Observatorio cultural, 15, 4-10. https://bit.ly/2JIKlpy

Carrasco, A., \& Fromm, G. (2016). How local market pressures shape leadership practices: evidence from Chile. Journal of Educational Administration and History, 48(4), 290-308. https://doi.org/10.1080/00220620.2016.1210584

Carrasco, A., Gutiérrez, G., \& Flores, C. (2017). Failed regulations and school composition: selective admission practices in Chilean primary schools. Journal of Education Policy, 32(5), 642-672. https://doi.org/10.1080/02680939.2 017.1312549

Carvalho, M.L., \& Normand, R. (2018). Introduction. In R. Normand, M. Liu, L. Carvalho, D. Andrade, \& L. Levasseur (Eds.), Education Policies and the Restructuring of the Educational Profession, Global and Comparative Perspectives (pp. 1-12). Springer.

Cavieres, E., \& Apple, M. (2016). La ley docente y la clase media: Controlando el desarrollo de los profesores chilenos. Cadernos CEDES, 36(100), 265-280. https://dx.doi.org/10.1590/cc0101-32622016171391

Chan, W. (2010). A Review of Education Reform-New Senior Secondary (NSS) Education in Hong Kong. International Education Studies, 3(4), 26-35. https://doi.org/10.5539/ies.v3n4p26

Coll, C. (2016). La personalización del aprendizaje escolar. El qué, el por qué y el cómo de un reto insoslayable. En J. Vilalta (Dir.), Reptes de l'educació a Catalunya. Anuari d'Educació 2015 (pp. 43-104). Fundació Jaume Bofill.
Cornejo, R., Albornoz, N., Castañeda, L., Palacios, D. Etcheberrigaray, G., Fernández, R., Gómez, S., Hidalgo, F., \& Lagos, J.I. (2015). Las prescripciones del trabajo docente en el nuevo marco regulatorio de políticas educativas en Chile. Psicoperspectivas, 14(2), 72-83. http://dx.doi.org/10.5027/PSICOPERSPECTIVASVOL14-ISSUE2-FULLTEXT-580

Darling-Hammond, L. (2014). Standards, assessments and educational policy: pursuit of genuine accountability. Educational Testing Service.

Falabella, A. (2015). El mercado escolar en Chile y el surgimiento de la Nueva Gestión Pública: el tejido de la política entre la dictadura neoliberal y los gobiernos de la centroizquierda (1979 a 2009). Educaçao \& Sociedade, 36(132), 699-722. http://dx.doi.org/10.1590/ES0101-73302015152420

Fardella, C. (2013). Resistencias cotidianas en torno a la institucionalización del modelo neoliberal en las políticas educacionales: El caso de la docencia en Chile. Psicoperspectivas, 12(2), 83-92. http://dx.doi.org/10.5027/psicoperspectivas-Vol12Issue2-fulltext-294

Fernández, R., Albornoz, N., Cornejo, R., \& Etcheberrigaray, G. (2016). Los discursos sobre autonomía del trabajo docente en el nuevo marco regulatorio educativo chileno. Currículo sem Fronteiras, 16(2), 283-302. https://bit.ly/33CcO7u

Fundación Chile (2013). Análisis de la implementación de los programas de integración escolar (PIE) en establecimientos que han incorporado estudiantes con necesidades educativas especiales transitorias (NEET). https://bit.ly/33CcV2U

Gleeson, D., \& Knights, D. (2015). Challenging dualism: Public professionalism in 'troubled' times. Sociology, 2(40), 277-295. https://doi.org/10.1177\%2F0038038506062033

Hall, D., Grimaldi, E., Gunter, H., Møller, J., Serpieri, R., \& Skedsmo, G. (2015). Educational reform and modernisation in Europe: The role of national contexts in mediating the new public management. European Educational Research Journal, 14(6), 487-507. https://doi.org/10.1177/1474904115615357

Herr, K. (2015). Cultivating disruptive subjectivities: Interrupting the new professionalism. 
Education Policy Analysis Archives, 23(86). http://dx.doi.org/10.14507/epaa.v23.2097

Holloway, J., Sørensen, T.B., \& Verger, A. (2017). Global perspectives on high-stakes teacher accountability policies: An introduction. Education Policy Analysis Archives, 25(85). http://dx.doi.org/10.14507/epaa.25.3325

Internacional de la Educación para América Latina (2015). La estandarización de la evaluación. Las pruebas nacionales e internacionales ¿medición o evaluación? Utdannings Forbundet/ Internacional de la Educación para América Latina.

Ley $\mathrm{N}^{\circ}$ 20.529. Sistema Nacional de Aseguramiento de la Calidad de la Educación Parvularia, Básica y Media y su Fiscalización. Diario Oficial de la República de Chile, Santiago, Chile, 27 de agosto de 2011.

Maroy, C., \& Pons, X. (2019). Accountability Policies in Education. A Comparative and Multilevel Analysis in France and Quebec. Springer.

Maroy, C., Pons, X., \& Dupuy, C. (2016). Vernacular globalisations: neo-statist accountability policies in France and Quebec education. Journal of Education Policy, 32(1), 100-122. https://doi.org/10.1080/02680939.2016.1239841

Meyer, A., Rose, D.H., \& Gordon, D. (2014). Universal Design for Learning: Theory and Practice. CAST.

Mintzberg, H. (1980). Structure in 5's: A synthesis of the research on organization design. Management Science, 26(3), 322-341. https://doi.org/10.1287/mnsc.26.3.322

Mintzberg, H. (1984). La estructuración de las organizaciones. Ariel Economía.

Mintzberg, H. (1992). El poder en la organización. Ariel Economía.

Mintzberg, H. (1993). El proceso estratégico. Conceptos, contextos y casos. Prentice-Hall Hispanoamericana.

Montecinos, C., Ahumada, L., Galdames, S., Campos, F., \& Leiva, M.V. (2015). Targets, threats and (dis)trust: The managerial troika for public school principals in Chile. Education Policy Analysis Archives, 23(87). http://dx.doi.org/10.14507/epaa.v23.2083

Luengo-Navas, J., \& Saura-Casanova, G. (2013). La performatividad en la educación. La construcción del nuevo docente y el nuevo gestor performativo. REICE-Revista Iberoamericana sobre Calidad, Eficacia y Cambio en Educación, 11(3), 139-153. https://bit.ly/2I1 epvQ

Normand, R., Liu, M., Carvalho, L.M., Andrade, D., \& LeVasseur, L. (2018). Education Policies and the Restructuring of the Educational Profession. Global and Comparative Perspectives. Springer.

Organización para la Cooperación y el Desarrollo Económicos (OCDE) (2017). Evaluaciones de políticas nacionales de educación. Educación en Chile. OCDE, Fundación SM.

Oyarzún, C., Soto, R., \& Moreno, K. (2019). Tensiones del trabajo docente en un contexto gerencialista: una revisión de literatura sobre el caso chileno. Educação (UFSM), 44(1).

http://dx.doi.org/10.5902/19846444434888

Parcerisa, L., \& Falabella, A. (2017). La consolidación del Estado evaluador a través de políticas de rendición de cuentas: Trayectoria, producción $\mathrm{y}$ tensiones en el sistema educativo chileno. Education Policy Analysis Archives, 25(89). http://dx.doi.org/10.14507/epaa.25.3177

Philpott, C. (2018). Teacher agency and professional learning communities: what can learning rounds in Scotland teach us? In M. Peters, B. Cowie, \& I. Menter (Eds.), A companion to research in teacher education (pp. 269-282). Springer.

Pino, M., Oyarzún, G., \& Salinas, I. (2016). Crítica a la estandarización para la rendición de cuentas: narrativas de resistencia para transformar el sistema de evaluación en Chile. Cadernos Cedes, 36(100), 337-354. https://doi.org/10.1590/cc0101-32622016171362

Pucheu, A. (2013). Lo que cambia son las personas. RIL Editores.

Reyes, I., \& Akkari, A. (2017). La privatización de la educación en Chile: análisis de los discursos del profesorado y de la dirección de los centros sobre la calidad de la educación y la rendición de cuentas. RASE, Revista de Sociología de la Educación, 10(3), 363-380. http://dx.doi.org/10.7203/RASE.10.3.9891

Rojas, M.T., \& Leyton, D. (2014). La nueva subjetividad docente: Construcción de subjetividades docentes en los inicios de la implementación de la Subvención Escolar Preferencial en 
Chile. Estudios pedagógicos, 40 (número especial), 205-221.

http://dx.doi.org/10.4067/S0718-07052014000200012

Rosas, R., \& Santa Cruz, C. (2013). Dime en qué colegio estudiaste y te diré qué CI tienes. Radiografía al desigual acceso al capital cognitivo en Chile. Ediciones UC.

Ruffinelli, A. (2016). Ley de desarrollo profesional docente en Chile: de la precarización sistemática a los logros, avances y desafíos pendientes para la profesionalización. Estudios Pedagógicos, 42(4), 261-279. https://dx.doi. org/10.4067/S0718-07052016000500015

Schmidt, T. (2006). A review of structure in fives: Designing effective organizations. https://bit.ly/3onM4j7

Sisto, V. (2012). Identidades desafiadas: individualización, managerialismo y trabajo docente en el Chile actual. PSYKHE, 21(2), 35-46. http://dx.doi.org/10.7764/psykhe.21.2.542

Taut, S., \& Sun, Y. (2014). The Development and Implementation of a National, Standardsbased, Multi-method Teacher Performance Assessment System in Chile. Education Policy Analysis Archives, 22(71). http://dx.doi.org/10.14507/epaa.v22n71.2014

Tsang, K.K., \& Kwong, T.L. (2016). Teachers' emotions in the context of education reform: labor process theory and social constructionism. British Journal of Sociology of Education, 38(6), 841-855.

https://doi.org/10.1080/01425692.2016.1182007
Verger, A., \& Normand, R. (2015). Nueva gestión pública y educación: Elementos teóricos y conceptuales para el estudio de un modelo de reforma educativa global. Educaçao \& Sociedade, 36(132), 599-622. http://dx.doi.org/10.1590/ES0101-73302015152799

Verger, A., Zancajo, A., \& Fontdevila, C. (2016). La economía política de la privatización educativa: políticas, tendencias y trayectorias desde una perspectiva. Revista Colombiana de Educación, (70), 47-78. https://doi.org/10.17227/01203916.70rce47.78

Verger, A., Moschetti, M., \& Fontdevila, C. (2017). La privatización educativa en América Latina: Una cartografía de políticas, tendencias y trayectorias. Education International. https://doi.org/10.13140/RG.2.2.16049.58724

Vieira, L., \& Oliveira, T. (2013). As condições do trabalho docente na Educação Infantil no Brasil: alguns resultados de pesquisa (20022012). Revista Educação em Questão, 46(32), 131-154. https://doi.org/10.21680/1981-1802.2013v46n32ID5125 Weinstein, J., Marfán, J., Horn, A., \& Muñoz, G. (2016). Chile: School leadership challenged by double accountability towards schools. In J. Easley II, \& P. Tulowitzki (Eds.), Educational Accountability. International perspectives on challenges and possibilities (pp. 54-72). Routledge. 